\title{
Radial Probe Endobronchial Ultrasound Using Guide Sheath-Guided Transbronchial Lung Biopsy in Peripheral Pulmonary Lesions without Fluoroscopy
}

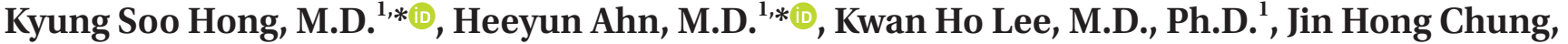 \\ M.D., Ph.D. ${ }^{\text {, }}$ Kyeong-Cheol Shin, M.D., Ph.D. ${ }^{1}$, Hyun Jung Jin, M.D., Ph.D. ${ }^{1}$, Jong Geol Jang, M.D. ${ }^{1}$, \\ Seok Soo Lee, M.D., M.S. ${ }^{2}$, Min Hye Jang, M.D., Ph.D. ${ }^{3}$ and June Hong Ahn, M.D., Ph.D. ${ }^{1}$ (i) \\ ${ }^{1}$ Division of Pulmonology and Allergy, Department of Internal Medicine, Respiratory Center, Yeungnam University Medical \\ Center, Yeungnam University College of Medicine, Daegu, ${ }^{2}$ Department of Thoracic and Cardiovascular Surgery, Yeungnam \\ University Medical Center, Yeungnam University College of Medicine, Daegu, ${ }^{3}$ Department of Pathology, Yeungnam University \\ College of Medicine, Daegu, Republic of Korea
}

Background: Radial probe endobronchial ultrasound-guided transbronchial lung biopsy (RP-EBUS-TBLB) has improved the diagnostic yield of bronchoscopic biopsy of peripheral pulmonary lesions (PPLs). The diagnostic yield and complications of RP-EBUS-TBLB for PPLs vary depending on the technique, such as using a guide sheath (GS) or fluoroscopy. In this study, we investigated the utility of RP-EBUS-TBLB using a GS without fluoroscopy for diagnosing PPLs.

Methods: We retrospectively reviewed data from 607 patients who underwent RP-EBUS of PPLs from January 2019 to July 2020. TBLB was performed using RP-EBUS with a GS without fluoroscopy. The diagnostic yield and complications were assessed. Multivariable logistic regression analyses were used to identify factors affecting the diagnostic yields.

Results: The overall diagnostic accuracy was $76.1 \%$ (462/607). In multivariable analyses, the size of the lesion $(\geq 20$ $\mathrm{mm}$; odds ratio $[\mathrm{OR}], 2.06 ; 95 \%$ confidence interval $[\mathrm{CI}], 1.27-3.33 ; \mathrm{p}=0.003)$, positive bronchus sign in chest computed tomography (OR, 2.30; 95\% CI, 1.40-3.78; $\mathrm{p}=0.001$ ), a solid lesion (OR, 2.40; 95\% CI, 1.31-4.41; $\mathrm{p}=0.005)$, and an EBUS image with the probe within the lesion (OR, 6.98; 95\% CI, 4.38-11.12; $\mathrm{p}<0.001)$ were associated with diagnostic success. Pneumothorax occurred in 2.0\% (12/607) of cases and chest tube insertion was required in $0.5 \%$ (3/607) of patients.

Conclusion: RP-EBUS-TBLB using a GS without fluoroscopy is a highly accurate diagnostic method in diagnosing PPLs that does not involve radiation exposure and has acceptable complication rates.

Keywords: Bronchoscopy; Diagnosis; Peripheral; Ultrasonography

Address for correspondence: June Hong Ahn, M.D., Ph.D.

Division of Pulmonology and Allergy, Department of Internal Medicine, Respiratory Center, Yeungnam University Medical Center, Yeungnam University College of Medicine, 170 Hyeonchung-ro, Nam-gu, Daegu 42415, Republic of Korea

Phone: 82-53-640-6577, Fax: 82-53-620-3849, E-mail: fireajh@gmail.com

*Kyung Soo Hong and Heeyun Ahn contributed equally to this work.

Received: Jan. 2, 2021, Revised: Mar. 24, 2021, Accepted: Jun. 23, 2021, Published online: Jun. 24, 2021

@(c) it is identical to the Creative Commons Attribution Non-Commercial License (http://creativecommons.org/licenses/by-nc/4.0/) 


\section{Introduction}

Bronchoscopy has evolved over the past few decades and endobronchial ultrasound (EBUS) is widely used in clinical practice. Among EBUS techniques, radial probe (RP) EBUSguided biopsy is commonly used to diagnose peripheral pulmonary lesions (PPLs) ${ }^{1}$.

This method offers high diagnostic yield and low complication rates for the diagnosis of PPLs. A meta-analysis that applied this method investigated 57 studies with a total of 7,872 PPLs and reported an overall weighted diagnostic yield of $70.6 \%$ (95\% confidence interval [CI], 68\%-73.1\%). The overall complication rate was $2.8 \%^{2}$. A more recent meta-analysis updated these results and reported a pooled sensitivity of 0.72 and complication rate of $0.7 \%$; sensitivity varied among institutions $^{3}$. The method of RP-EBUS-guided transbronchial lung biopsy (RP-EBUS-TBLB) varies significantly among institutions regarding the use of additional guidance tools such as a guide sheath (GS) or fluoroscopy ${ }^{2-4}$.

The GS technique provides access to target bronchial lesions for repeated sampling and protecting against bleeding from the biopsy site by wedging the GS into the target lesion ${ }^{5}$. The use of fluoroscopic guidance in addition to RP-EBUS can improve diagnostic yield while administering acceptable radiation doses to patients and clinicians ${ }^{6-8}$. However, fluoroscopy still exposes patients and practitioners to radiation, and consumes additional space, manpower, and costs (e.g., to install a shield room $)^{9}$.

Few studies have explored the efficacy and safety of RPEBUS-TBLB using a GS without fluoroscopy for PPLs ${ }^{10-13}$. Hence, in this study, we evaluated the diagnostic yield and complications of RP-EBUS-TBLB in diagnosing PPLs and identified factors associated with the diagnostic yield.

\section{Materials and Methods}

\section{Study design and subjects}

We performed a retrospective observational study on 607 consecutive patients who underwent RP-EBUS for PPLs from January 2019 to July 2020 at Yeungnam University Hospital (a 930-bed, university-affiliated, tertiary referral hospital in
Daegu, South Korea). All EBUS-visualized lesions ( $\mathrm{n}=537$ ) were biopsied via RP-EBUS-TBLB (Figure 1).

\section{CT and bronchoscopy}

All patients underwent thin-section chest computed tomography (CT) $(0.75 \mathrm{~mm}$ slice thickness at intervals of 0.75 mm; SOMATOM Definition AS 64-slice CT system, Siemens Healthcare, Erlangen, Germany) less than seven days before RP-EBUS. Experienced pulmonologists reviewed the chest CT images before the procedure and planned a bronchial route to access the target lesion. The bronchus sign in CT was defined as the presence of a bronchus leading to the target lesion. The distance from the lesion to the pleura was measured as the shortest distance on an axial plane CT scan, as described previously ${ }^{14}$.

All bronchoscopy procedures were performed by three pulmonologists, each with more than 5 years of experience in respiratory medicine. Patients were sedated with $2.5-5.0$ $\mathrm{mg}$ of intravenous midazolam and 25-50 $\mu \mathrm{g}$ fentanyl. A 4 mm bronchoscope (BF P260F, Olympus, Tokyo, Japan) was used to reach the bronchus closest to the target lesion. Then, a RP-EBUS (UM S20-17S, Olympus) was inserted inside a GS through the bronchoscope working channel. Following the discovery of the PPL, the RP was then removed, leaving the GS in place. Then, bronchial brush and biopsy forceps were introduced into the GS and brushings and biopsy specimens were collected. When TBLB was performed at our hospital, the lesion was identified with the RP, performed gain on the first three lung tissue samples, re-inserted the RP to ensure that the GS was not re-positioned in the lesion, and then performed an extra biopsy. X-ray fluoroscopy was not used.

Figure 2 shows representative cases of RP-EBUS-TBLB with GS and without fluoroscopy in patients with suspected lung cancer.

\section{Diagnostic classification}

A final diagnosis of malignancy was made based on the definite histological evidence of malignancy, or clinical features consistent with malignancy. Benign lung lesion was diagnosed according to the following criteria: identification of definite benign features, regression of the lesion with medical

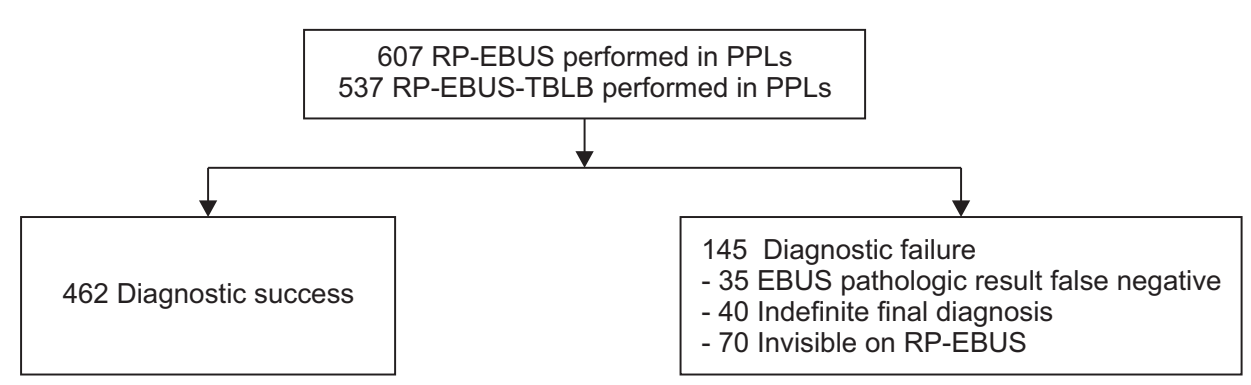

Figure 1. Study flowchart. PPLs: peripheral pulmonary lesions; RP-EBUS-TBLB: radial probe endobronchial ultrasoundguided transbronchial lung biopsy. 

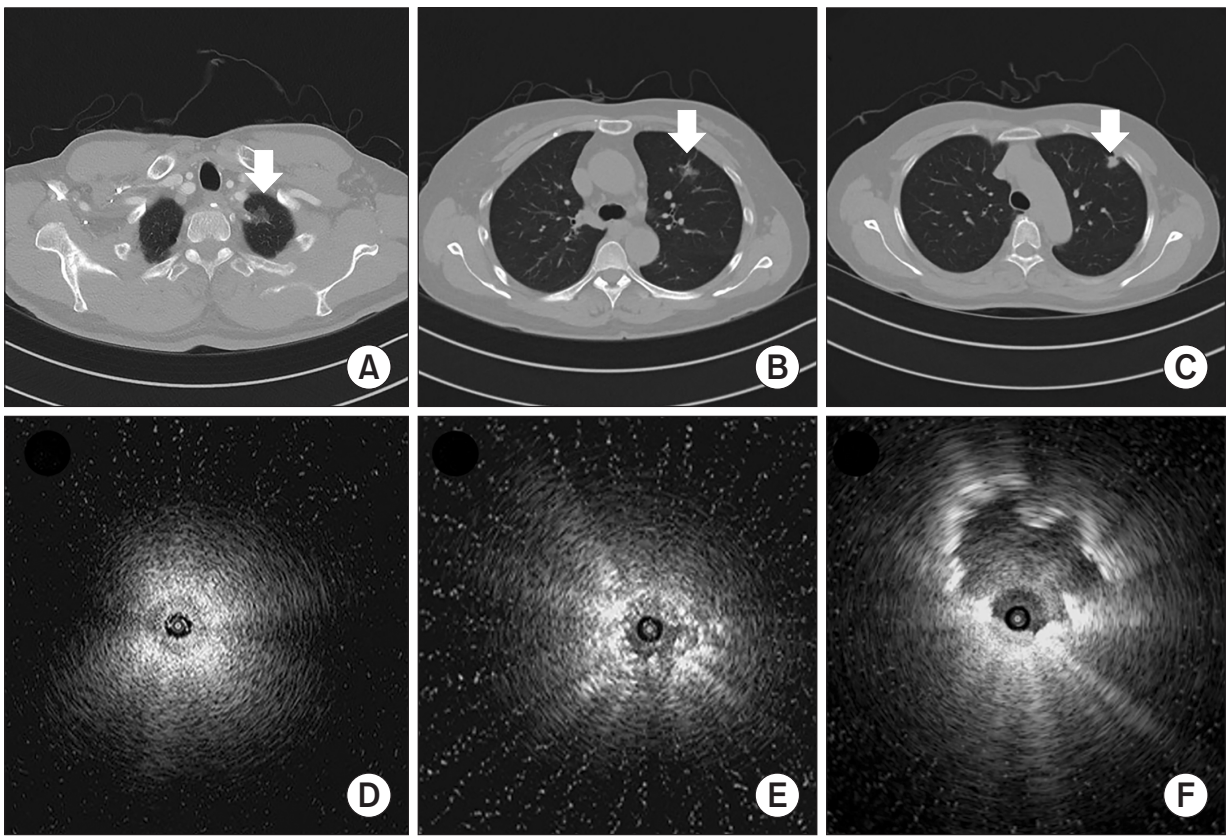

Figure 2. Representative cases. (A) Computed tomography (CT) scan showing a $20 \mathrm{~mm}$ ground glass nodule (arrow). (B) CT scan showing a $16 \mathrm{~mm}$ part-solid nodule (arrow). (C) CT scan showing a $15 \mathrm{~mm}$ speculated subpleural solid nodule (arrow). (D) Radial probe endobronchial ultrasound (RP-EBUS) shows a subtle increase in the intensity and radius of the whitish acoustic shadow, known as the blizzard sign, after which biopsy was performed and adenocarcinoma was diagnosed. (E) RP-EBUS shows diffuse heterogeneity with several hyperechoic dots (the mixed-blizzard sign), after which biopsy was performed and adenocarcinoma was diagnosed. (F) RP-EBUS in the anterior segment of the left upper lobe showing an EBUS image containing the probe and biopsy revealed adenocarcinoma. treatment, and a stable size for at least 12 months. Lung lesion that was neither benign nor malignant was defined as indefinite. Lesions diagnosed as benign both at the beginning and at the end were considered as true-negative. Lesions initially diagnosed as benign but finally diagnosed with malignancy (EBUS pathologic result false-negative) were designated as false-negative. Indefinite final diagnosis and invisible on RPEBUS cases were also considered as false-negative.

\section{Statistical analyses}

Continuous variables were compared to Student's t test or the Mann-Whitney U test and were expressed as means \pm standard deviations. Categorical variables were compared using chi-square or Fisher exact tests and were described as frequencies (percentages). We calculated the diagnostic yield by dividing the number of diagnostic successes by the total number of cases. To explore factors that affected diagnostic yield, the study population was divided into two groups: a diagnostic success group (true-positive and true-negative results) and a diagnostic failure group (false-positive, and falsenegative results). Univariable and multivariable (using the factors with $\mathrm{p}<0.1$ in univariable analyses) logistic regression analyses were performed to identify factors affecting the diagnostic yields. In all analyses, $\mathrm{p}<0.05$ under a two-tailed test was considered as statistically significant. All statistical procedures were performed using SPSS version 24.0 (IBM Corp., Armonk, NY, USA).

\section{Ethics statement}

This study was conducted in accordance with the tenets of the Declaration of Helsinki, and its protocol was reviewed and approved by the institutional review board of Yeungnam University Hospital (YUH IRB 2020-09-025). The requirement for informed consent was waived because of the retrospective study design.

\section{Results}

\section{Baseline characteristics}

The baseline characteristics of the 607 patients according to diagnostic success status are presented in Table 1. Their mean age was $67.8 \pm 12.0$ years and 395 patients were men (65.1\%). Chronic obstructive pulmonary disease (COPD; $\mathrm{n}=167,27.5 \%$ ) was the most common underlying lung disease, followed by old pulmonary tuberculosis $(n=41,6.8 \%)$, asthma $(n=16,2.6 \%)$, idiopathic pulmonary fibrosis $(n=12,2.0 \%)$, combined pulmonary fibrosis and emphysema $(n=6,1.0 \%)$, and pneumoconiosis $(n=6,1.0 \%)$. The mean percent of predicted forced expiratory volume in 1 second was $83.0 \% \pm 19.5 \%$. The most common location of the lung lesion was the right upper lobe ( $\mathrm{n}=172,28.3 \%)$, and the mean distance from the pleura to the lung lesion was $13.4 \pm 13.2 \mathrm{~mm}$. The PPLs were significantly larger in the diagnostic success group than in the diagnostic failure group $(32.8 \pm 16.2 \mathrm{~mm}$ vs. $23.5 \pm 13.7 \mathrm{~mm}, \mathrm{p}<0.001)$. The lung lesions were classified as solid ( $\mathrm{n}=355,58.5 \%)$, part-solid $(n=53,8.7 \%)$, ground-glass opacity $(n=24,4.0 \%)$, cavity $(n=71$, 
Table 1. Baseline characteristics of the patients according to diagnostic success status

\begin{tabular}{|c|c|c|c|c|}
\hline Characteristic & Total $(n=607)$ & $\begin{array}{l}\text { Diagnostic success } \\
\qquad(n=462)\end{array}$ & $\begin{array}{l}\text { Diagnostic failure } \\
\quad(n=145)\end{array}$ & p-value \\
\hline \multicolumn{5}{|l|}{ Patients } \\
\hline Age, yr & $67.8 \pm 12.0$ & $68.2 \pm 12.0$ & $66.6 \pm 11.8$ & 0.170 \\
\hline Male sex & $395(65.1)$ & $300(64.9)$ & $95(65.5)$ & 0.898 \\
\hline Underlying lung disease & & & & 0.579 \\
\hline COPD & $167(27.5)$ & $126(27.3)$ & $41(28.3)$ & \\
\hline Asthma & $16(2.6)$ & $14(3.0)$ & $2(1.4)$ & \\
\hline IPF & $12(2.0)$ & $11(2.4)$ & $1(0.7)$ & \\
\hline CPFE & $6(1.0)$ & $6(1.3)$ & $0(0)$ & \\
\hline Old pulmonary TB & $41(6.8)$ & $33(7.1)$ & $8(5.5)$ & \\
\hline Pneumoconiosis & $6(1.0)$ & $5(1.1)$ & $1(0.7)$ & \\
\hline \multicolumn{5}{|l|}{ Pulmonary function test, $\%$} \\
\hline $\mathrm{FEV}_{1}$ & $83.0 \pm 19.5$ & $82.2 \pm 18.9$ & $85.7 \pm 20.9$ & 0.061 \\
\hline FVC & $82.0 \pm 16.8$ & $81.1 \pm 16.7$ & $84.9 \pm 17.2$ & 0.017 \\
\hline $\mathrm{FEV}_{1} / \mathrm{FVC}$ & $73.2 \pm 11.4$ & $73.2 \pm 11.3$ & $73.3 \pm 11.8$ & 0.927 \\
\hline \multicolumn{5}{|l|}{ Lung lesions } \\
\hline Location & & & & 0.820 \\
\hline RUL & $172(28.3)$ & $130(28.1)$ & $42(29.0)$ & \\
\hline RML & $32(5.3)$ & $24(5.2)$ & $8(5.5)$ & \\
\hline RLL & $151(24.9)$ & $119(25.8)$ & $32(22.1)$ & \\
\hline LUL & $161(26.5)$ & $118(25.5)$ & $43(29.6)$ & \\
\hline LLL & $91(15.0)$ & $71(15.4)$ & $20(13.8)$ & \\
\hline Distance from pleura (mm) & $13.4 \pm 13.2$ & $13.3 \pm 13.2$ & $13.8 \pm 13.2$ & 0.691 \\
\hline Size $(\mathrm{mm})$ & $31.0 \pm 16.1$ & $32.8 \pm 16.2$ & $23.5 \pm 13.7$ & $<0.001$ \\
\hline Characteristics & & & & $<0.001$ \\
\hline Solid & $355(58.5)$ & $276(59.7)$ & $79(54.5)$ & \\
\hline Part-solid & $53(8.7)$ & $32(6.9)$ & $21(14.5)$ & \\
\hline Ground-glass opacity & $24(4.0)$ & $11(2.4)$ & $13(9.0)$ & \\
\hline Cavity & $71(11.7)$ & $54(11.7)$ & $17(11.7)$ & \\
\hline Consolidation & $104(17.1)$ & 89 (19.3) & $15(10.3)$ & \\
\hline Bronchus sign in CT & & & & $<0.001$ \\
\hline Positive & $476(78.4)$ & $399(86.4)$ & $77(53.1)$ & \\
\hline Negative & $131(21.6)$ & $63(13.6)$ & $68(46.9)$ & \\
\hline \multicolumn{5}{|l|}{ Procedure } \\
\hline EBUS image & & & & $<0.001$ \\
\hline Within & $425(70.0)$ & $380(82.3)$ & $45(31.0)$ & \\
\hline Adjacent & $112(18.5)$ & $82(17.7)$ & $30(20.7)$ & \\
\hline Invisible & $70(11.5)$ & $0(0.0)$ & $70(48.3)$ & \\
\hline No. of forceps biopsies per lesion* $(\mathrm{n}=537)$ & $5.1 \pm 1.8$ & $5.3 \pm 1.7$ & $4.0 \pm 2.1$ & $<0.001$ \\
\hline Procedure time, min & $18.2 \pm 8.1$ & $19.3 \pm 7.9$ & $17.4 \pm 8.1$ & $<0.001$ \\
\hline
\end{tabular}

Values are presented as mean \pm standard deviation or number (\%).

*Number excluding lesions that failed to perform biopsies due to invisible case.

COPD: chronic obstructive pulmonary disease; IPF: idiopathic pulmonary fibrosis; CPFE: combined pulmonary fibrosis and emphysema; TB: tuberculosis; $\mathrm{FEV}_{1}$ : forced expiratory volume in one second; FVC: forced vital capacity; RUL: right upper lobe; RML: right middle lobe; RLL: right lower lobe; LUL: left upper lobe; LLL: left lower lobe; CT: computed tomography; EBUS: endobronchial ultrasound. 
$11.7 \%)$, and consolidation ( $\mathrm{n}=104,17.1 \%)$. An EBUS image with the probe within the lesion was observed considerably more frequently in the diagnostic success group (82.3\% vs. $31.0 \%, \mathrm{p}<0.001)$. The mean procedure time was $18.2 \pm 8.1 \mathrm{~min}$ utes.

\section{Pathologic results and diagnostic performance}

The initial pathologic results and final diagnosis are listed in Table 2. Out of the 607 lung lesions, 373 (61.4\%) were diagnosed as malignant, $178(29.3 \%)$ as benign, and $56(9.2 \%)$ as indefinite. Among the malignant lesions ( $\mathrm{n}=373), 304$ (81.5\%) were diagnosed by RP-EBUS-TBLB. Lesions with false-negatives $(n=69)$ were diagnosed as malignant by percutaneous transthoracic needle biopsy ( $\mathrm{n}=36)$, surgical resection $(\mathrm{n}=20)$,
EBUS-guided transbronchial needle aspiration $(\mathrm{n}=11)$, and bone biopsy $(\mathrm{n}=2)$. Adenocarcinoma of the lung $(\mathrm{n}=228$, $61.1 \%$ ) was the most common diagnosis among definitive malignant diagnoses. Among the benign lesions, pneumonia $(\mathrm{n}=79,44.4 \%)$ was the most common diagnosis among definitive benign features. The diagnostic performance of RP-EBUSTBLB according to the size of the PPLs is summarized in Table 3 . The sensitivity, specificity, positive predictive value, negative predictive value, and diagnostic accuracy of RP-EBUS-TBLB were $67.8 \%$ (305/450), 100\% (157/157), 100\% (305/305), $52.0 \%$ (157/302), and 76.1\% (462/607), respectively. The diagnostic performance of RP-EBUS-TBLB was best for PPLs $>30$ mm group ( $87.7 \%)$, followed by the $20-30 \mathrm{~mm}$ group ( $78.2 \%$ ) and $<20$ mm group (54.7\%).

Table 2. Initial pathologic results and final diagnosis

\begin{tabular}{|c|c|c|}
\hline & Initial pathologic results $(n=607)$ & Final diagnosis $(n=607)$ \\
\hline Malignant & 304 & 373 \\
\hline Adenocarcinoma & 194 & 228 \\
\hline Adenosquamous cell carcinoma & 3 & 3 \\
\hline Squamous cell carcinoma & 57 & 72 \\
\hline NSCLC, NOS & 31 & 38 \\
\hline SCLC & 9 & 11 \\
\hline Metastatic carcinoma & 6 & 15 \\
\hline Neuroendocrine tumor & 1 & 3 \\
\hline Malignant lymphoma & 3 & 3 \\
\hline Benign & 233 & 178 \\
\hline Pneumonia & 16 & 79 \\
\hline Pulmonary TB & 11 & 45 \\
\hline Nontuberculous mycobacteria & 4 & 18 \\
\hline Aspergillosis & 4 & 5 \\
\hline Actinomycosis & 1 & 2 \\
\hline Cryptococcosis & 1 & 1 \\
\hline Chondroid hamartoma & 1 & 3 \\
\hline Nonspecific benign features & 88 & 25 \\
\hline Negative for malignant cell & 44 & - \\
\hline Atypical pneumocytes & 10 & - \\
\hline Chronic granulomatous inflammation & 21 & - \\
\hline Anthracosis & 11 & - \\
\hline Total necrosis & 2 & - \\
\hline Chronic inflammation & 126 & - \\
\hline No definitive diagnosis & - & 56 \\
\hline Invisible on EBUS & 70 & - \\
\hline
\end{tabular}

NSCLC: non-small cell lung cancer; NOS: not otherwise specified; SCLC: small cell lung cancer; TB: tuberculosis; EBUS: endobronchial ultrasound. 
Table 3. Diagnostic performance of RP-EBUS-TBLB according to the size of PPLs

\begin{tabular}{|c|c|c|c|c|}
\hline Parameter & $\begin{array}{l}<20 \mathrm{~mm} \\
(\mathrm{n}=159)\end{array}$ & $\begin{array}{c}20-30 \mathrm{~mm} \\
(\mathrm{n}=188)\end{array}$ & $\begin{array}{l}>30 \mathrm{~mm} \\
(\mathrm{n}=260)\end{array}$ & $\begin{array}{l}\text { Diagnostic performance } \\
\qquad(n=607)\end{array}$ \\
\hline True-positive, $\mathrm{n}$ & 54 & 102 & 149 & 305 \\
\hline True-negative, $\mathrm{n}$ & 33 & 45 & 79 & 157 \\
\hline False-positive, $\mathrm{n}$ & 0 & 0 & 0 & 0 \\
\hline False-negative, $\mathrm{n}$ & 72 & 41 & 32 & 145 \\
\hline EBUS pathologic result false negative, $n$ & 15 & 12 & 8 & 35 \\
\hline Indefinite final diagnosis, $\mathrm{n}$ & 19 & 8 & 13 & 40 \\
\hline Invisible on EBUS & 38 & 21 & 11 & 70 \\
\hline Sensitivity, \% & 42.9 & 71.3 & 82.3 & 67.8 \\
\hline Specificity, \% & 100.0 & 100.0 & 100.0 & 100.0 \\
\hline PPV, \% & 100.0 & 100.0 & 100.0 & 100.0 \\
\hline NPV, $\%$ & 31.4 & 52.3 & 71.2 & 52.0 \\
\hline Diagnostic accuracy & 54.7 & 78.2 & 87.7 & 76.1 \\
\hline
\end{tabular}

RP-EBUS-TBLB: radial probe endobronchial ultrasound-guided transbronchial lung biopsy; PPL: peripheral pulmonary lesion; PPV: positive predictive value; NPV: negative predictive value.

Table 4. Factors affecting diagnostic success of RP-EBUS-TBLB using a GS without fluoroscopy for diagnosing PPLs

\begin{tabular}{|c|c|c|c|c|c|c|}
\hline & \multirow[b]{2}{*}{$\begin{array}{c}\text { Diagnostic } \\
\text { success } \\
(n=462)\end{array}$} & \multirow[b]{2}{*}{$\begin{array}{c}\text { Diagnostic } \\
\text { failure } \\
(n=145)\end{array}$} & \multicolumn{2}{|c|}{ Univariable analyses } & \multicolumn{2}{|c|}{ Multivariable analyses } \\
\hline & & & $\begin{array}{c}\text { Odds ratio } \\
\text { (95\% confidence } \\
\text { interval) }\end{array}$ & p-value & $\begin{array}{c}\text { Odds ratio } \\
\text { (95\% confidence } \\
\text { interval })\end{array}$ & p-value \\
\hline Age, yr & $68.2 \pm 12.0$ & $66.6 \pm 11.8$ & $1.01(0.99-1.03)$ & 0.170 & - & - \\
\hline Male sex & $300(64.9)$ & $95(65.5)$ & $1.03(0.69-1.52)$ & 0.898 & - & - \\
\hline Size, $\mathrm{mm}$ & $32.8 \pm 16.2$ & $23.5 \pm 13.7$ & $1.05(1.04-1.07)$ & $<0.001$ & - & - \\
\hline$\geq 20$ & $375(81.2)$ & $73(50.3)$ & $4.25(2.85-6.35)$ & $<0.001$ & $2.06(1.27-3.33)$ & 0.003 \\
\hline$<20$ & $87(18.8)$ & $72(49.7)$ & 1.00 & - & - & - \\
\hline Bronchus sign in chest $\mathrm{CT}$ & - & - & - & - & - & - \\
\hline Positive & $399(86.4)$ & $77(53.1)$ & $5.59(3.67-8.52)$ & $<0.001$ & $2.30(1.40-3.78)$ & 0.001 \\
\hline Negative & $63(13.6)$ & $68(46.9)$ & 1.00 & - & - & - \\
\hline \multicolumn{7}{|l|}{ Characteristic } \\
\hline Solid & $419(90.7)$ & $111(76.6)$ & $3.00(1.82-4.90)$ & $<0.001$ & $2.40(1.31-4.41)$ & 0.005 \\
\hline Non-solid & $43(9.3)$ & $34(23.4)$ & 1.00 & - & - & - \\
\hline \multicolumn{7}{|l|}{ EBUS image } \\
\hline Within & $380(82.3)$ & $45(31.0)$ & $10.30(6.73-15.75)$ & $<0.001$ & $6.98(4.38-11.12)$ & $<0.001$ \\
\hline $\begin{array}{l}\text { Adjacent to the lesion } \\
\text { or outside }\end{array}$ & $82(17.7)$ & $100(69.0)$ & 1.00 & - & - & - \\
\hline
\end{tabular}

Values are presented as mean \pm standard deviation or number (\%) unless otherwise indicated.

RP-EBUS-TBLB: radial probe endobronchial ultrasound-guided transbronchial lung biopsy; GS: guide sheath; PPL: peripheral pulmonary lesion; CT: computed tomography; Solid: solid+cavity+consolidation; Non-solid: part solid+ground-glass opacity.

\section{Factors affecting diagnostic success}

In patients with definitive final diagnoses, we investigated factors affecting diagnostic success (Table 4). Univariable analyses revealed that larger size $(\geq 20 \mathrm{~mm}$; odds ratio $[\mathrm{OR}]$, $4.25 ; 95 \% \mathrm{CI}, 2.85-6.35 ; \mathrm{p}<0.001)$, positive bronchus sign in chest CT (OR, 5.59; 95\% CI, 3.67-8.52; $\mathrm{p}<0.001$ ), a solid lesion (OR, 3.00; 95\% CI, 1.82-4.90; $\mathrm{p}<0.001$ ), and an EBUS image 
with the probe within the lesion (OR, 10.30; 95\% CI, 6.73-15.75 $\mathrm{p}<0.001)$ were significantly associated with diagnostic success. Based on multivariable analyses, larger size $(\geq 20 \mathrm{~mm}$; OR, 2.06; 95\% CI, 1.27-3.33; $\mathrm{p}=0.003$ ), positive bronchus sign in chest CT (OR, 2.30; 95\% CI, 1.40-3.78; $\mathrm{p}=0.001$ ), a solid lesion (OR, 2.40; 95\% CI, 1.31-4.41; $\mathrm{p}=0.005)$, and an EBUS image with the probe within the lesion (OR, 6.98; 95\% CI, 4.38-11.12; $\mathrm{p}<0.001$ ) were independent factors affecting diagnostic success.

\section{Complications}

Among the 607 patients who underwent RP-EBUS, there were 12 cases of pneumothorax (2.0\%). Nine patients (1.5\%) recovered with oxygen therapy and three $(0.5 \%)$ required chest tube insertion.

\section{Discussion}

This study confirms that RP-EBUS-TBLB using a GS without fluoroscopy is a highly safe diagnostic method in patients with PPLs. The sensitivity, specificity, positive predictive value, negative predictive value, and diagnostic accuracy of RP-EBUSTBLB were $67.8 \%, 100 \%, 100 \%, 52.0 \%$, and $76.1 \%$, respectively. The diagnostic performance of RP-EBUS-TBLB was better in larger PPLs. Larger lesions ( $\geq 20 \mathrm{~mm}$ ), positive bronchus sign in chest CT, a solid lesion, and an EBUS image with the probe within the lesion were independent factors affecting diagnostic success. Pneumothorax occurred in $2.0 \%$ of patients, and $0.5 \%$ required chest tube insertion. To the best of our knowledge, this is the largest study to have analyzed the utility of RPEBUS-TBLB using a GS without fluoroscopy.

Kurimoto et al. ${ }^{5}$ developed a technique for EBUS using a GS, and mentioned that EBUS-GS is a useful method for collecting samples from PPLs even when the lesions are too small to be detectable under fluoroscopy. In PPLs $\leq 20 \mathrm{~mm}$, TBLB with GS showed a higher diagnostic sensitivity than the TBLB without GS for the diagnosis of PPLs ${ }^{15}$. In addition, fluoroscopy was not helpful to confirm whether the forceps were within the lesion, and the diagnostic yield was the same regardless of the use of fluoroscopy for PPLs $\leq 20 \mathrm{~mm}$. Advantages of the GS technique are that it provides access to bronchial lesions for repeated sampling and protects against bleeding from the biopsy site by wedging the GS into the bronchial lumen ${ }^{5}$. In diagnosing PPLs $\leq 20 \mathrm{~mm}$, the diagnostic yield of our study (using a GS without fluoroscopy) was $54.7 \%$, comparable to other studies using a GS with fluoroscopy ${ }^{16-19}$. In our experience, if GS is appropriately used, a high diagnostic rate can be expected if accurate localization and EBUS image findings are achieved, even if fluoroscopy is not used.

Regarding fluoroscopy, it may be combined with TBLB to confirm whether the forceps are within the lesion. Fluoroscopy can be more helpful in performing biopsy at lower lobe lesions with a high probability of re-positioning of GS due to respiration or coughing. As described in the methods section, the lesion was identified with the RP, perform gain on the first three lung tissue samples, re-inserted the RP to ensure that the GS was not re-positioned in the lesion, and then performed an extra biopsy. Using this approach, we addressed the disadvantages of not using fluoroscopy. As a result, the diagnostic success rate of the right lower lobe $(78.8 \%, 119 / 151)$ and left lower lobe $(78.0 \%, 71 / 91)$ was not different from the rate of other lobes, as shown in Table 1. It is important to improve the diagnosis of PPLs and reduce radiation exposure during the examination. Fluoroscopy has disadvantages, including excessive radiation exposure for patients and practitioners. In addition, fluoroscopy consumes additional space, manpower, and cost for installing a shield room. Thus, in institutions where fluoroscopy is difficult to use, RP-EBUS-TBLB using a GS without fluoroscopy can be considered as a useful method for diagnosing PPLs.

A few studies have explored the efficacy and safety of RPEBUS-TBLB using a GS without fluoroscopy for PPLs. Yoshikawa et al. ${ }^{10}$ were the first to publish the outcomes of RPEBUS-TBLB in 123 PPLs using a GS without fluoroscopy. A total of $61.8 \%$ of PPLs were diagnosed, and the diagnostic yield for PPLs $>20 \mathrm{~mm}$ (75.6\%) was higher than PPLs $\leq 20 \mathrm{~mm}$ (29.7\%). Lesions $>2 \mathrm{~cm}$ and the location (middle lobe and the lingular segment) of the PPLs were independent predictors of diagnostic success ${ }^{10}$. It can be estimated that our research is a little superior in terms of diagnostic accuracy. The diagnostic accuracy was significantly different in PPLs smaller than $3 \mathrm{~cm}$. The difference in baseline characteristics of PPLs between two studies may have affected the results. There was no difference in size of PPLs between the two studies. However, positive bronchus sign in chest CT was higher in our study (476 of 607 lesions, $78.5 \%$ ) compared to the previous study (76 of 123 lesions, $61.8 \%$ ). The high percentage of solid lesion in our study (87.3\%) compared to the previous study $(67.0 \%)$ may have also affected the diagnostic accuracy. Eberhardt et al. ${ }^{11}$ conducted a randomized trial of multimodality diagnostic arms; in subgroup analyses, a diagnostic yield of $69 \%$ was achieved in EBUS-GS without fluoroscopy in 39 PPLs. Minami et al. ${ }^{15}$ reported that the diagnostic sensitivity of 60 PPLs with EBUS-GS was $83.3 \%$. Minezawa et al. ${ }^{12}$ analyzed 149 PPLs who underwent EBUS-GS without fluoroscopy for small PPLs $(\leq 30 \mathrm{~mm})$ and a total diagnostic yield of $72.5 \%$ was reported. CT bronchus sign positive was an independent factor associated with diagnostic success. Zhu et al. ${ }^{13}$ reported diagnosis rate of $64.0 \%$ for EBUS-GS without fluoroscopy among 150 PPLs. Our study analyzed 607 PPLs and the diagnostic accuracy was $76.1 \%$. As reported in previous studies, lesions $\geq 20 \mathrm{~mm}$, positive bronchus sign in chest CT, a solid lesion, and EBUS probe within the lesion were independent factors of diagnostic success.

Complication rates of RP-EBUS-TBLB were low in previous studies and most of the complications were small pneumo- 
thorax and minimal bleeding problems. One meta-analysis revealed that the overall complications were $2.8 \%$, and chest tube insertion was required in $0.2 \%$ of cases $^{2}$. One observational study focusing on complications of RP-EBUS-TBLB in 965 PPLs showed overall complication rates of 1.3\%. Pneumothorax occurred in $0.8 \%$ of patients, and $0.3 \%$ of the patients required chest tube drainage ${ }^{20}$. Our study demonstrated that pneumothorax occurred in 12 patients $(2.0 \%)$ and chest tube insertion was required in three patients $(0.5 \%)$. The relatively high incidence of pneumothorax compared to other studies is thought to be due to the high proportion of COPD patients $(27.8 \%)$. Indeed, all three patients who required chest tube insertion were COPD patients with emphysema.

This study had several limitations. First, since it was a retrospective study conducted at a single center with PPLs, the results cannot be generalized, and selection bias cannot be excluded. However, the pulmonologists performed RP-EBUS as a first-choice biopsy modality in their everyday routine practice, not just in selected patients. Second, although our patients were followed-up for at least 12 months, there were 56 patients who still had nodules with an indefinite diagnosis. Although most previously published articles in the fields of RPEBUS excluded PPLs with an indefinite diagnosis to calculate diagnostic yield, our study included indefinite diagnosis cases in the calculation of diagnostic yield. Given the high diagnostic yield of RP-EBUS-TBLB with GS without fluoroscopy and the acceptable rates of complications, our study showed that it can be performed in routine clinical settings for diagnosing PPLs without fluoroscopy. Moreover, our results highlighted the important role of RP-EBUS in diagnosing PPLs, which required early diagnosis.

In conclusion, RP-EBUS-TBLB using a GS without fluoroscopy is a highly accurate diagnostic method without exposure to radiation and with acceptable complication rates for diagnosing PPLs. Lesions $\geq 20 \mathrm{~mm}$, positive bronchus sign in chest $\mathrm{CT}$, a solid lesion, and having the probe within the lesion were important for diagnostic success.

\section{Authors' Contributions}

Conceptualization: Hong KS, Ahn H, Jang JG, Ahn JH. Data curation: Hong KS, Ahn H, Jang MH, Jang JG, Ahn JH. Investigation: Hong KS, Ahn H, Jang JG, Ahn JH. Supervision: Hong KS, Ahn H, Lee KH, Chung JH, Shin KC, Jin HJ, Lee SS, Jang JG, Ahn JH. Writing-original draft: Hong KS, Ahn JH. Writingreview \& editing: Ahn JH.

\section{Conflicts of Interest}

No potential conflict of interest relevant to this article was reported.

\section{Funding}

This research was supported by the Bio \& Medical Technology Development Program of the National Research Foundation (NRF) funded by the Ministry of Science \& ICT (grant number 2021M3E5D1A0201526521 and 2021R1C1C100950811).

\section{References}

1. Ahn JH. An update on the role of bronchoscopy in the diagnosis of pulmonary disease. Yeungnam Univ J Med 2020;37:25361.

2. Ali MS, Trick W, Mba BI, Mohananey D, Sethi J, Musani AI. Radial endobronchial ultrasound for the diagnosis of peripheral pulmonary lesions: a systematic review and metaanalysis. Respirology 2017;22:443-53.

3. Sainz Zuniga PV, Vakil E, Molina S, Bassett RL Jr, Ost DE. Sensitivity of radial endobronchial ultrasound-guided bronchoscopy for lung vancer in patients with peripheral pulmonary lesions: an updated meta-analysis. Chest 2020;157:994-1011.

4. Steinfort DP, Khor YH, Manser RL, Irving LB. Radial probe endobronchial ultrasound for the diagnosis of peripheral lung cancer: systematic review and meta-analysis. Eur Respir J 2011;37:902-10.

5. Kurimoto N, Miyazawa T, Okimasa S, Maeda A, Oiwa H, Miyazu Y, et al. Endobronchial ultrasonography using a guide sheath increases the ability to diagnose peripheral pulmonary lesions endoscopically. Chest 2004;126:959-65.

6. Steinfort DP, Einsiedel P, Irving LB. Radiation dose to patients and clinicians during fluoroscopically-guided biopsy of peripheral pulmonary lesions. Respir Care 2010;55:1469-74.

7. Lee SC, Kim EY, Chang J, Lee SH, Han CH. Diagnostic value of the combined use of radial probe endobronchial ultrasound and transbronchial biopsy in lung cancer. Thorac Cancer 2020;11:1533-40.

8. Casutt A, Prella M, Beigelman-Aubry C, Fitting JW, Nicod L, Koutsokera A, et al. Fluoroscopic-guided radial endobronchial ultrasound without guide sheath for peripheral pulmonary lesions: a safe and efficient combination. Arch Bronconeumol 2015;51:338-43.

9. Lee K. New wine into new wineskins: radial endobronchial ultrasound for parenchymal lung infiltration. Tuberc Respir Dis 2019;82:264-5.

10. Yoshikawa M, Sukoh N, Yamazaki K, Kanazawa K, Fukumoto S, Harada M, et al. Diagnostic value of endobronchial ultrasonography with a guide sheath for peripheral pulmonary lesions without X-ray fluoroscopy. Chest 2007;131:1788-93.

11. Eberhardt R, Anantham D, Ernst A, Feller-Kopman D, Herth F. Multimodality bronchoscopic diagnosis of peripheral lung lesions: a randomized controlled trial. Am J Respir Crit Care Med 2007;176:36-41. 
12. Minezawa T, Okamura T, Yatsuya $H$, Yamamoto N, Morikawa $\mathrm{S}$, Yamaguchi T, et al. Bronchus sign on thin-section computed tomography is a powerful predictive factor for successful transbronchial biopsy using endobronchial ultrasound with a guide sheath for small peripheral lung lesions: a retrospective observational study. BMC Med Imaging 2015;15:21.

13. Zhu J, Tang F, Gu Y. A prospective study on the diagnosis of peripheral lung cancer using endobronchial ultrasonography with a guide sheath and computed tomography-guided transthoracic needle aspiration. Ther Adv Med Oncol 2018;10: 1758834017752269.

14. Tay JH, Irving L, Antippa P, Steinfort DP. Radial probe endobronchial ultrasound: factors influencing visualization yield of peripheral pulmonary lesions. Respirology 2013;18:185-90.

15. Minami D, Takigawa N, Morichika D, Kubo T, Ohashi K, Sato A, et al. Endobronchial ultrasound-guided transbronchial biopsy with or without a guide sheath for diagnosis of lung cancer. Respir Investig 2015;53:93-7.

16. Kikuchi E, Yamazaki K, Sukoh N, Kikuchi J, Asahina H, Imura
M, et al. Endobronchial ultrasonography with guide-sheath for peripheral pulmonary lesions. Eur Respir J 2004;24:533-7.

17. Asahina H, Yamazaki K, Onodera Y, Kikuchi E, Shinagawa N, Asano F, et al. Transbronchial biopsy using endobronchial ultrasonography with a guide sheath and virtual bronchoscopic navigation. Chest 2005;128:1761-5.

18. Boonsarngsuk V, Kanoksil W, Laungdamerongchai S. Comparison of diagnostic performances among bronchoscopic sampling techniques in the diagnosis of peripheral pulmonary lesions. J Thorac Dis 2015;7:697-703.

19. Chen A, Chenna P, Loiselle A, Massoni J, Mayse M, Misselhorn D. Radial probe endobronchial ultrasound for peripheral pulmonary lesions: a 5-year institutional experience. Ann Am Thorac Soc 2014;11:578-82.

20. Hayama M, Izumo T, Matsumoto Y, Chavez C, Tsuchida T, Sasada S. Complications with endobronchial ultrasound with a guide sheath for the diagnosis of peripheral pulmonary lesions. Respiration 2015;90:129-35. 